\title{
Síndrome de Mirizzi tipo V: Manejo laparoscópico
}

\author{
Gonzalo Andrés Domínguez Alvarado a, ; Daniela D’vera Camargo ${ }^{\text {b,1; }}$; Iván David Lozada Martínez ${ }^{\mathrm{c}, 2}$;
} Felipe López Ramírez ${ }^{\text {d,3 }}$; Luis Ernesto López Gómez ${ }^{\mathrm{e}, 1}$

\begin{abstract}
${ }^{a}$ Médico. Especialista en epidemiología clínica.Profesor, Director Grupo de investigación e innovación quirúrgica. Fundador y Coordinador Semillero de Innovación e Investigación en Cirugía (SIIC - UNAB). ORCID: https://orcid.org/0000-0002-7512-8733, gdominguez@ unab.edu.co ; b Médica Interna, ORCID: https://orcid.org/0000-0002-4901-7490, dd@unab.edu.co ; ' Estudiante de Medicina, ORCID: https://orcid.org/0000-0002-1960-7334, ivandavidloma@gmail. com; ${ }^{\mathrm{d}}$ Ingeniero biomédico. Médico. Investigador en “The Institute for Cancer Care at Mercy Medical Center”. ORCID: https://orcid.org/0000-0002-1560-9172, if.lopez718@uniandes.edu.co ; ${ }^{\mathbf{e}}$ Médico. Especialista en Cirugía General y Oncológica. Fellowship en obesidad, Gastro Obeso Center de São Paulo. ORCID:

https://orcid.org/0000-0002-3666-2159, cirugia@unab.edu.co

1. Universidad Autónoma de Bucaramanga 2. Universidad de Cartagena. 3. Universidad de los Andes.
\end{abstract} DOI: https://doi.org/10.22517/25395203.24657

\section{Resumen}

Introducción: El Síndrome de Mirizzi es una complicación infrecuente de la enfermedad litiásica biliar, con una incidencia menor al $1 \%$ en países desarrollados, puede desarrollarse en cinco variantes, siendo menos frecuente la variante tipo V. La literatura actual discrepa sobre el manejo de esta condición, afirmando que la cirugía laparoscópica no es segura como procedimiento estándar.

Caso Clínico: Se presenta el caso de Síndrome de Mirizzi en un hombre de 80 años, que es remitido al departamento de urgencias por sospecha de sepsis de origen abdominal, con estudio ecográfico de colelitiasis, neumobilia y dilatación de las vías biliares. Se realizó Colangiopancreatografía retrógrada endoscópica con imposibilidad técnica para la movilización y extracción de los cálculos por gran tamaño, recurriéndose a exploración a través de técnica laparoscópica, obteniéndose resultados satisfactorios.

Conclusión: Para tratar el Síndrome de Mirizzi, es necesario considerar las características del paciente y la experiencia del cirujano ya que ambos factores influyen directamente en la modalidad del tratamiento, sus complicaciones y tasas de éxito. En el presente caso, la experiencia del autor principal en el manejo de procedimientos mínimamente invasivos y la consideración de reducir el riesgo de complicaciones como infecciones en un paciente frágil, fueron los factores que influyeron para la decisión de intervención laparoscópica.

Palabras clave: Síndrome de Mirizzi, Colelitiasis, Colangitis, Laparoscopia.

\section{Mirizzi syndrome type V: laparoscopic management}

\begin{abstract}
Introduction: Mirizzi's syndrome is an infrequent complication of biliary lithiasic disease, with an incidence of less than $1 \%$ in developed countries, being even less frequent the type $\mathrm{V}$ variant. Current literature disagrees on the management of this condition, stating that laparoscopic surgery is not safe as a standard procedure.
\end{abstract}

Clinical Case: We present the case of Mirizzi's Syndrome in an 80-year-old man, which is referred to the emergency department for suspicion of sepsis of abdominal origin, with ultrasound study of cholelithiasis, pneumoobilia and dilation of the bile ducts. Retrograde endoscopic cholangiopancreatography was performed with technical impossibility for the mobilization and extraction of large-size stones, resorting to exploration through laparoscopic technique, obtaining satisfactory results.

Conclusion: It is necessary to emphasize that the type of Mirizzi syndrome, the patient's characteristics and the surgeon's experience directly influence the treatment modality, its complications and/or success rates. In the present case, the experience of the main author in the management of minimally invasive procedures and the consideration of reducing the risk of complications such as infections in a fragile patient, were the factors that influenced the decision for laparoscopic intervention.

Key words: Mirizzi Syndrome, Cholelithiasis, Cholangitis, Laparoscopy. 


\section{Introducción}

El Síndrome de Mirizzi (SM), conocido también como síndrome de compresión biliar extrínseca, fue descrito por primera vez en 1948 por el cirujano argentino Pablo Luis Mirizzi como una complicación poco frecuente de la enfermedad litiásica biliar (1). Este síndrome se caracteriza por la compresión extrínseca del conducto biliar, producida por la presión aplicada indirectamente por uno o varios cálculos incrustados en el infundíbulo de la vesícula biliar (bolsa de Hartmann) o conducto cístico, dando como resultado una obstrucción parcial o completa del conducto hepático común, que puede finalizar en una disfunción hepática (1),(3). A su vez, la inflamación crónica resultante y la ulceración puede desencadenar fístulas internas desde la vesícula biliar hasta el conducto hepático común, el colédoco y el duodeno (2),(4).

El SM es una patología de presentación infrecuente, con una incidencia menor al 1\% por año en los países occidentales desarrollados, y del 4.7\%-5.7\% en los países en vía de desarrollo (3). En Colombia, se ha reportado prevalencias de alrededor del $4 \%$ (5). Esta entidad no posee signos patognomónicos, sin embargo, se ha encontrado que la ictericia obstructiva es la presentación más común (50-100\%), y que frecuentemente se acompaña de dolor en el hipocondrio derecho o dolor epigástrico y en algunos casos con fiebre, náuseas, vómitos, coluria, escalofríos, taquicardia y anorexia (1),(2),(6),(7).

A lo largo del tiempo, se han descrito varias clasificaciones para este síndrome, actualmente, la más utilizada es la modificada por Csendes et al (8) en el 2007, y validada por Beltrán et al (9) quienes establecen cinco tipos (Tabla 1).

\begin{tabular}{|c|c|c|c|c|}
\hline Tipo Mirizzi & Definición & Clínica & Diagnóstico & Tratamiento \\
\hline Tipo I & $\begin{array}{l}\text { Compresión externa del con- } \\
\text { ducto biliar por un cálculo } \\
\text { impactado en el infundíbulo } \\
\text { de la vesicular biliar o el con- } \\
\text { ducto cístico }\end{array}$ & $\begin{array}{l}\text { Ictericia, dolor abdominal, náu- } \\
\text { seas, vómitos, orina oscura. }\end{array}$ & $\begin{array}{l}\text { Ultrasonografía abdominal, } \\
\text { TAC, colangioresonancia mag- } \\
\text { nética, CPRE, diagnóstico in- } \\
\text { traoperatorio. }\end{array}$ & $\begin{array}{l}\text { Colecistectomía fundoquistica con co- } \\
\text { langiografía sin exploración de las vía } \\
\text { biliar y extracción de cálculos biliares. }\end{array}$ \\
\hline Tipo II & $\begin{array}{l}\text { Fístula bilio-enterica que } \\
\text { afecta menos de un tercio de } \\
\text { la circunferencia del conducto } \\
\text { biliar }\end{array}$ & $\begin{array}{l}\text { Ictericia, dolor abdominal, náu- } \\
\text { seas, vómitos, orina oscura. }\end{array}$ & $\begin{array}{l}\text { Ultrasonografía abdominal, } \\
\text { TAC, colangioresonancia mag- } \\
\text { nética, CPRE, diagnóstico in- } \\
\text { traoperatorio. }\end{array}$ & $\begin{array}{l}\text { (I) Colecistectomía subtotal; (II) Ex- } \\
\text { tracción de cálculos, sutura del conduc- } \\
\text { to biliar y colocación de tubo en T; (III) } \\
\text { Y de Roux Hepato-yeyunostomía; (IV) } \\
\text { Colecisto-colédoco-duodenostomia; } \\
\text { (V) Colecisto-colédoco-yeyunostomía. }\end{array}$ \\
\hline Tipo II & $\begin{array}{l}\text { Fístula bilio-enterica que } \\
\text { abarca hasta dos tercios de la } \\
\text { circunferencia del conducto. }\end{array}$ & $\begin{array}{l}\text { Ictericia, dolor abdominal, náu- } \\
\text { seas, vómitos, orina oscura. }\end{array}$ & $\begin{array}{l}\text { Ultrasonografía abdominal, } \\
\text { TAC, colangioresonancia mag- } \\
\text { nética, CPRE, diagnostico in- } \\
\text { traoperatorio. }\end{array}$ & $\begin{array}{l}\text { (I) Colecistectomía subtotal; (II) Ex- } \\
\text { tracción de cálculos, sutura del conduc- } \\
\text { to biliar y colocación de tubo en T; (III) } \\
\text { Y de Roux Hepato-yeyunostomía; (IV) } \\
\text { Colecisto-colédoco-duodenostomia }\end{array}$ \\
\hline Tipo IV & $\begin{array}{l}\text { Fístula bilio-enterica con obs- } \\
\text { trucción completa del conduc- } \\
\text { to biliar. }\end{array}$ & $\begin{array}{l}\text { Ictericia, dolor abdominal, náu- } \\
\text { seas, vómitos, orina oscura. }\end{array}$ & $\begin{array}{l}\text { Ultrasonografía abdominal, } \\
\text { TAC, colangioresonancia mag- } \\
\text { nética, CPRE, diagnostico in- } \\
\text { traoperatorio. }\end{array}$ & $\begin{array}{l}\text { (I) Colecistectómica subtotal; (II) } \\
\text { Anastomosis bilioenterica duodenal; } \\
\text { (III) Y de Roux Hepato-yeyunostomía }\end{array}$ \\
\hline Tipo Va & $\begin{array}{l}\text { Incluye la presencia de una } \\
\text { fistula bilio-enterica junto con } \\
\text { cualquier otro Mirizzi }\end{array}$ & $\begin{array}{l}\text { Ictericia, dolor abdominal, náu- } \\
\text { seas, vómitos, orina oscura. }\end{array}$ & $\begin{array}{l}\text { Ultrasonografía abdominal, } \\
\text { TAC, colangioresonancia mag- } \\
\text { nética, CPRE, diagnostico in- } \\
\text { traoperatorio. }\end{array}$ & $\begin{array}{l}\text { (I) División y sutura simple de fistulas } \\
\text { bilioentericas em las vísceras involu- } \\
\text { cradas; (II) Colecistectomía total o sub- } \\
\text { total según la presencia de una fistula } \\
\text { colecistobiliar. }\end{array}$ \\
\hline Tipo Vb & $\begin{array}{l}\text { Incluye una fístula bilio-ente- } \\
\text { rica con íleo biliar }\end{array}$ & & & $\begin{array}{l}\text { (I) Tratamiento del íleo biliar; (II) En } \\
\text { segunda intención colecistectomía total } \\
\text { o subtotal. }\end{array}$ \\
\hline
\end{tabular}

Tabla 1: Clasificación del Síndrome Mirizzi

El diagnóstico preoperatorio de SM está dado por la clínica, pruebas de laboratorio e imágenes diagnósticas como la ultrasonografía abdominal, tomografía axial computarizada (TAC), Colangio Resonancia Magnética (CRMN) y Colangio-Pancreatografia Retrógrada Endoscópica (CPRE) (2). Dada la baja incidencia y por ende la poca experiencia, el diagnóstico preoperatorio no es fácil, existiendo rangos entre el 8 al 63,4\% de los casos.

La literatura actual discrepa sobre el manejo de esta condición, afirmando que la cirugía laparoscópica no es segura como 
procedimiento estándar, y mucho menos, en pacientes con enfermedad avanzada (10-12). Sin embargo, en los últimos años esta técnica se ha posicionado como una opción de tratamiento por sus excelentes resultados. El objetivo de este manuscrito consiste en compartir la experiencia en el manejo de Síndrome de Mirizzi tipo V mediante laparoscopía, sin complicaciones y con una recuperación satisfactoria.

\section{Presentación del caso}

Paciente masculino de 80 años de edad con antecedentes de Diabetes Mellitus tipo 2 e Hipertensión Arterial en metas, que ingresa al departamento de urgencias remitido por sospecha de sepsis de origen abdominal, con estudio ecográfico de colelitiasis, neumobilia y dilatación de las vías biliares. Al examen físico, se encuentra en regular condición general, estable hemodinámicamente, ictérico, colurico, con leve dolor a la palpación en hipocondrio derecho, sin signos de irritación peritoneal y sin déficit neurológico.

Se realizan paraclínicos de ingreso, donde se encuentra leucocitosis moderada y neutrofilia. Hiperbilirrubinemia a expensas de la bilirrubina directa y anemia normocítica normocrómica moderada. En base a los hallazgos, se establece diagnostico probable de colangitis grado III y colecistitis Grado II, con manifestaciones sugestivas de coledocolitiasis, por lo que se indica CRMN (Figura 1), observando severa dilatación de la vía biliar extrahepática y neumobilia, consistente con coledocolitiasis masiva. No se identifica vesícula biliar. En función de los hallazgos, se establece diagnóstico definitivo de coledocolitiasis.

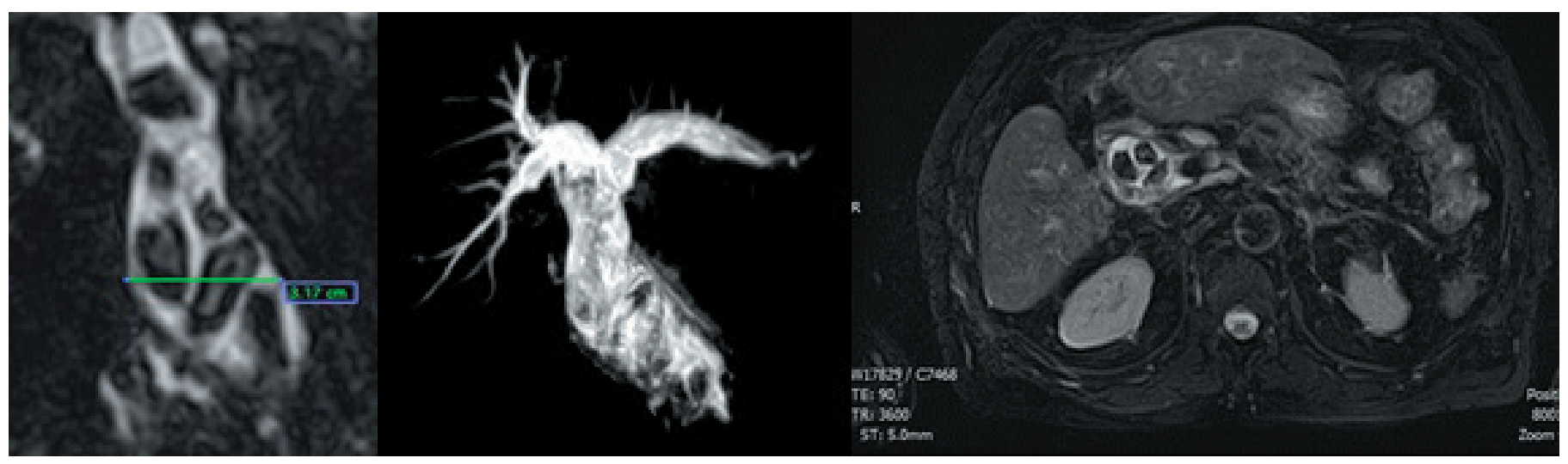

Figura 1. Dilatación de la vía biliar intra y extrahepática. El conducto hepático común y el colédoco se encuentran distendidos, a nivel intrapancreatico el colédoco alcanza un calibre de $27 \mathrm{~mm}$, al interior de los dos conductos se aprecian múltiples imágenes nodulares facetadas, con diámetros variables que oscilan entre 3 y $15 \mathrm{~mm}$. No se identificó la vesícula biliar. Hay cambios en la señal en el sistema ductal en el lóbulo izquierdo que sugiere neumobilia.

Se indica CPRE, llevándose a cabo con normalidad pero con imposibilidad técnica para la movilización y extracción de los cálculos por gran tamaño de estos. Debido al deterioro del cuadro clínico, se decide someter a exploración quirúrgica convencional de la vía biliar, optando por la técnica laparoscópica.

Durante la intervención, se identifica plastrón inflamatorio crónico que involucra el epiplón mayor, ángulo hepático del colon y el duodeno. El primer hallazgo mayor fue la confirmación de "ausencia de vesícula", encontrando en su lugar un pequeño remanente fibroso y atrófico, adherido a la vía biliar principal. El segundo hallazgo importante fue la gran dilatación del colédoco con diámetro de $3 \mathrm{~cm}$ y firmemente adherido a la segunda porción del duodeno. A este nivel se identificó la presencia de fístula colédoco-duodenal. Una vez efectuada la disección y separación de estos epitelios se procedió a efectuar sutura laparoscópica del duodeno. Posteriormente se procedió a efectuar coledocotomía longitudinal amplia con salida de material bilio-purulento.

Se identificaron y extrajeron múltiples cálculos facetados, midiendo el mayor de ellos $2 \mathrm{~cm}$ de diámetro. La exploración laparoscópica del colédoco cambiando la lente por una de $5 \mathrm{~mm}$ de diámetro y $30^{\circ}$, permitió observación proximal y distal hasta lograr la extracción de la totalidad de los cálculos. En esta maniobra se identificó stent biliar de previa 
colocación transduodenal endoscópica, dejándose en su lugar. Luego de lavado exhaustivo y exploración con sonda balón (Fogarty), se procedió a colocación de tubo de drenaje biliar (Tubo en $\mathrm{T}$ de Kher) (Figura 2), y cierre de coledocotomía con sutura laparoscópica intra-corpórea de polidoxanona calibre 3/0 (Figura 3). El tiempo operatorio fue de dos horas y media, con sangrado mínimo.

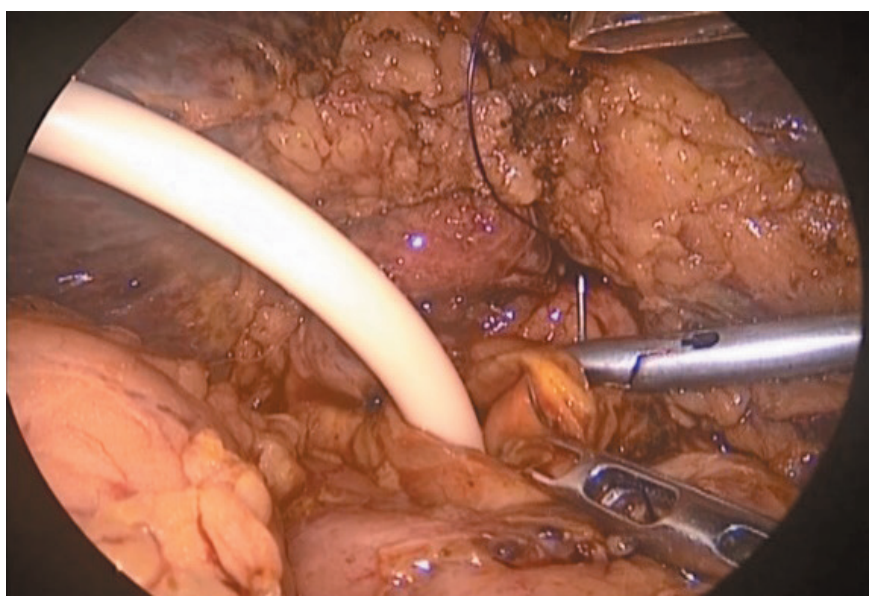

Figura 2: Fotografía intraoperatoria. Colocación de tubo

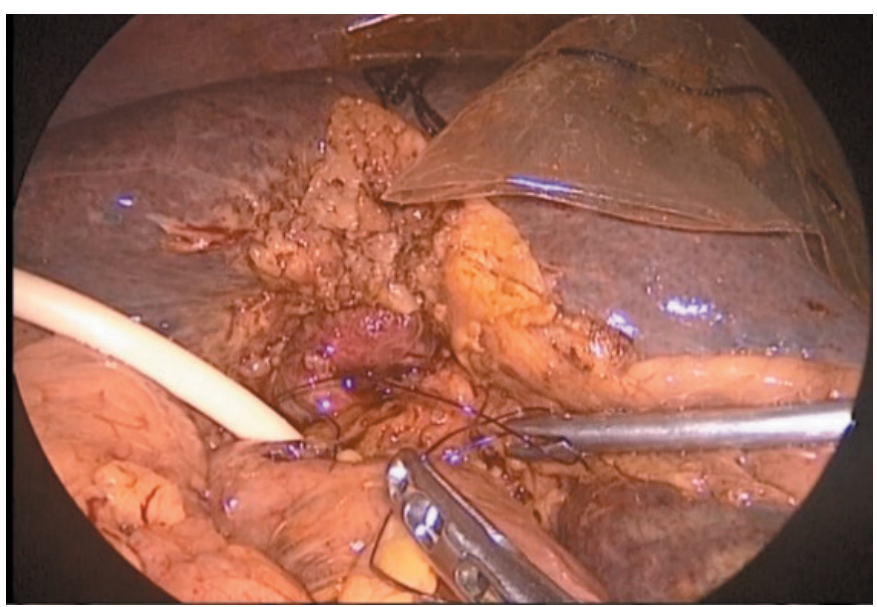

Figura 3: Fotografía intraoperatoria. Cierre de coledocotomía con sutura laparoscópica intra corpórea de polidoxanona calibre 3/0.

Durante el postoperatorio el paciente presenta íleo adinámico que resolvió espontáneamente. Al observar evolución satisfactoria, se da de alta hospitalaria a los cinco días. A las dos semanas se retira tubo de drenaje biliar, y en el control a las cuatro semanas, se evidencia resolución completa del cuadro sin complicaciones.

\section{Discusión}

La baja incidencia de SM a nivel global y la presentación del cuadro biliar inespecífico, constituye un reto para el diagnóstico precoz y tratamiento eficaz. Hoy por hoy, el estudio inicial ante la sospecha de enfermedad biliar es la ultrasonografía abdominal. A través de este medio no invasivo y de bajo costo, se pueden identificar cálculos biliares y colecistitis. En muy pocos casos, el hallazgo de una vesícula atrófica y un conducto hepático común ectasico con un colédoco integro, sugiere la presencia de SM. Sin embargo, esta prueba tiene tan solo un $29 \%$ de precisión diagnóstica y una sensibilidad entre el $8.3 \%$ $27 \%$ (13).

Por otra parte, la CRMN es un estudio de alta sensibilidad y especificidad en la detección de litios y estenosis de la vía biliar, ya que permite evidenciar el estrechamiento del colédoco, la presencia de un litio en el conducto cístico y la dilatación de la vía intra y extra hepática, además de la ilustración del árbol biliar, característica importante, que servirá como guía en la realización de procedimientos endoscópicos o quirúrgicos (14),(15). Actualmente el estándar de oro para el diagnóstico de SM son las imágenes aportadas por la CPRE. Técnica que permite la visualización amplia de los conductos biliares extrahepáticos, además de mostrar claramente la compresión extrínseca por cálculos biliares impactados, pudiendo determinar también con precisión la presencia y ubicación de fístulas (2). Tiene una sensibilidad del $76,2 \%$ e incluso algunos estudios refieren una sensibilidad del 100\% (4),(16). Igualmente, en múltiples ocasiones es utilizado como tratamiento inicial o complementario al procedimiento quirúrgico. No obstante, el diagnóstico de SM es identificado y confirmado en gran parte de los casos durante el intraoperatorio.

La solución definitiva del SM consiste en el tratamiento quirúrgico (2). Los cambios patológicos generan distorsión de la anatomía por adherencias densas como consecuencia de la inflamación crónica. Esto favorece el riesgo de fístula biliar interna, y por ende, mayor riesgo de lesión del conducto biliar o hemorragia masiva durante la disección del triángulo de Calot (16). Tradicionalmente, la cirugía abierta ha sido considerada como la técnica de elección para el tratamiento del SM (4). Esto como resultado de su relativa seguridad en comparación con la técnica por laparoscópica, la cual se asocia con altas tasas de conversión ( $31 \%$ - 100\%), y una mayor incidencia de lesiones de las vías biliares (16).

Actualmente este manejo es controversial, ya que el enfoque laparoscópico mínimamente invasivo tiene múltiples ventajas. Estancias hospitalarias más cortas, mejor uso de recursos, menor tasas de infección postoperatorias, entre otras (2). Algunos autores 
mencionan que la laparoscopía sólo es segura si se realiza en los pacientes con SM tipo I, sin embargo, Yuan et al (17) llevaron a cabo un estudio prospectivo, donde demostraron que la colecistectomía subtotal laparoscópica fue exitosa en todos los paciente con SM tipo II sin conversiones ni morbilidad, junto a una estancia hospitalaria más corta $(7.21 \pm 1.61$ vs $15,31 \pm$ $3,82$ días, $\mathrm{P}<0,01)$, menor pérdida de sangre (162.81 \pm 40.83 vs $207.55 \pm 37.01 \mathrm{~mL}, \mathrm{P}=0.425$ ), y sin diferencia significativa en la tasa de recurrencia $(4.55 \%$ vs $9.52 \%$, $\mathrm{P}=0.522$ ) de coledocolitiasis (17). Sin embargo, es poca la literatura que hable sobre la efectividad y seguridad de la laparoscopia en SM tipo III, IV y V (17-20).

Es necesario resaltar que el tipo de SM, las características del paciente y la experiencia del cirujano, influyen directamente en la modalidad del tratamiento, sus complicaciones y/o tasas de éxito. En el presente caso, la experiencia del autor principal en el manejo de procedimientos mínimamente invasivos y la consideración de reducir el riesgo de complicaciones como infecciones en un paciente frágil, fueron los factores que influyeron para la decisión de intervención laparoscópica. Para poder definir seguridad y eficacia de este laparoscopía vs cirugía abierta en el manejo de $\mathrm{SM}$, es necesario llevar a cabo estudios multicéntricos con el mayor nivel de evidencia, que permitan evaluar detalladamente diversas variables que puedan influir en los resultados peri y posoperatorios.

\section{Referencias}

1. Valderrama-Treviño AI, Granados-Romero JJ, Espejel-Deloiza M, Chernitzky-Camaño J, Barrera Mera B, Estrada-Mata AG, et al. Updates in Mirizzi syndrome. HepatoBiliary Surg Nutr. 2017; 6(3):170-8.

2. Jones MW, Ferguson T. Mirizzi Syndrome. 2021 Feb 8. In: StatPearls [Internet]. Treasure Island (FL): StatPearls Publishing; 2021 Jan-. PMID: 29494098.

3. Beltrán MA. Mirizzi syndrome: History, current knowledge and proposal of a simplified classification. World J Gastroenterol. 2012; 18(34):4639-50.

4. Chen H, Siwo EA, Khu M, Tian Y. Current trends in the management of Mirizzi Syndrome: A review of literature. Medicine (Baltimore). 2018 Jan;97(4):e9691. doi: 10.1097/MD.0000000000009691.

5. Rodríguez Carolina AG. El síndrome de compresión biliar extrínseca benigna o síndrome de Mirizzi: experiencia de cinco años en el Hospital de San José. Rev Colomb Cirugía. 2008; 23:6-11.

6. Lee KF. Mirizzi syndrome: a new approach to an old problem. Hepatobiliary Surg Nutr. 2018; 7(1):56-57.
7. Kimura J, Takata N, Lefor AK, Kanzaki M, Mizokami K. Laparoscopic subtotal cholecystectomy for Mirizzi syndrome: A report of a case. Int J Surg Case Rep. 2019; 55:32-34.

8. Csendes A, Muñoz C, Albán M. Síndrome de Mirizzi Fístula colecistobiliar, una nueva clasificación. Rev Chil Cir. 2007; 59(Suppl):63-64.

9. Beltran MA, Csendes A, Cruces KS. The relationship of Mirizzi syndrome and cholecystoenteric fistula: Validation of a modified classification. World J Surg. 2008; 32(10):2237-43.

10. Al-Akeely MHA, Alam MK, Bismar HA, Khalid K, Al-Teimi I, Al-Dossary NF. Mirizzi syndrome: Ten years experience from a teaching hospital in Riyadh. World J Surg. 2005; 29(12):1687-92.

11. Borz-Baba C, Levy DA, Cohen ME. PostCholecystectomy Mirizzi Syndrome: A Case Report and Review of the Literature. Am J Case Rep. 2019; 20:1290-1298.

12. Erben Y, Benavente-Chenhalls LA, Donohue JM, Que FG, Kendrick ML, Reid-Lombardo KM, et al. Diagnosis and treatment of Mirizzi syndrome: 23-year mayo clinic experience. J Am Coll Surg. 2011; 213(1):114-9.

13. Bo LI, Xun LI, Wen-ce Z, Ming-yan HE, Wenbo M, Lei Z, et al. Effect of endoscopic retrograde cholangiopancreatography combined. 2013; 126(18):3515-8.

14. Reverdito R, De Moricz A, De Campos T, Pacheco Júnior AM, Silva RA. Síndrome de Mirizzi graus III e IV: Tratamento cirúrgico. Rev Col Bras Cir. 2016; 43(4):243-7.

15. Yetişir F, Şarer AE, Acar HZ, Parlak O, Basaran B, Yazıcıoğlu O.Laparoscopic Resection of Cholecystocolic Fistula and Subtotal Cholecystectomy by Tri-Staple in a Type V Mirizzi Syndrome. Case Reports Hepatol. 2016; 2016:1-4.

16. Alemi F, Seiser N, Ayloo S. Gallstone Disease: Cholecystitis, Mirizzi Syndrome, Bouveret Syndrome, Gallstone Ileus. Surg Clin North Am. 2019; 99(2):231244.

17. Yuan H, Yuan T, Sun X, Zheng M. A Minimally Invasive Strategy for Mirizzi Syndrome Type II: Combined Endoscopic with Laparoscopic Approach. Surg Laparosc Endosc Percutaneous Tech. 2016; 26(3):248-52.

18. Xu XQ, Hong T, Li BL, Liu W, He XD, Zheng CJ. Mirizzi syndrome: Our experience with 27 cases in 
PUMC Hospital. Chinese Med Sci J. 2013; 28(3):172-7.

19. Clemente G, Tringali A, De Rose A, Panettieri E, Murazio M, Nuzzo G, et al. Mirizzi Syndrome: Diagnosis and Management of a Challenging Biliary Disease. Can J Gastroenterol Hepatol. 2018; 2018:6962090.

20. Seara Costa R, Vieira F, Costa JM, Ferreira A. Mirizzi syndrome: when the gallbladder meets bile ducts. Rev Esp Enferm Dig. 2019; 111(6):481-482. 